\title{
CONFORMALLY HOMEOMORPHIC LORENTZ SURFACES NEED NOT BE CONFORMALLY DIFFEOMORPHIC
}

\author{
ROBERT W. SMYTH AND TILLA WEINSTEIN
}

(Communicated by Christopher B. Croke)

\begin{abstract}
A Lorentz surface $\mathscr{L}$ is an ordered pair $(S,[h])$ where $S$ is an oriented $C^{\infty}$ 2-manifold and [h] the set of all $C^{\infty}$ metrics conformally equivalent to a fixed $C^{\infty}$ Lorentzian metric $h$ on $S$. (Thus Lorentz surfaces are the indefinite metric analogs of Riemann surfaces.) This paper describes subsets of the Minkowski 2-plane which are conformally homeomorphic, but not even $C^{1}$ conformally diffeomorphic. It also describes subsets of the Minkowski 2-plane which are $C^{j}$ but not $C^{j+1}$ conformally diffeomorphic for any fixed $j=1,2, \ldots$. Finally, the paper describes a Lorentz surface conformally homeomorphic to a subset of the Minkowski 2-plane, but not $C^{1}$ conformally diffeomorphic to any subset of the Minkowski 2-plane.
\end{abstract}

\section{INTRODUCTION}

A Lorentz surface is the indefinite metric analog of a Riemann surface. In a landmark paper [1], Kulkarni defined the conformal boundary of any simply connected surface with Lorentzian metric. The results in [1] made clear that Lorentz surfaces deserve a name of their own, reflecting the amazing similarities and contrasts of their properties with those of Riemann surfaces. To provide a proper context for the result announced in the title of this paper, some background is required. For a more thorough treatment of this material, see [3].

A Lorentz surface $\mathscr{L}$ is an ordered pair $(S,[h])$ where $S$ is an oriented $C^{\infty}$ surface and $[h]$ the set of all metrics on $S$ conformally equivalent to a fixed indefinite $C^{\infty}$ metric $h$ on $S$. Conformal equivalence $h \sim \hat{h}$ of two $C^{\infty}$ metrics $h$ and $\hat{h}$ on $S$ is taken in the strict sense that $\hat{h}=\lambda h$ for some positive $C^{\infty}$ function $\lambda$ on $S$. Thus, indefinite $C^{\infty}$ metrics are conformally equivalent iff they identify the same sets of spacelike, timelike and null vectors in each tangent space $S_{p}$.

Any indefinite $C^{\infty}$ metric $h$ on $S$ determines a pair of $C^{\infty}$ null direction fields $X, Y$ naturally ordered by requiring that arbitrarily small rotations in the positive sense of the direction $X_{p}$ (resp. $Y_{p}$ ) assigned by $X$ (resp. $Y$ ) at any point $p$ on $S$ take $X_{p}$ (resp. $Y_{p}$ ) to a spacelike (resp. timelike) position in

Received by the editors June 30, 1993 and, in revised form, April 5, 1994.

1991 Mathematics Subject Classification. Primary 53C50, 53A30.

(c) 1995 American Mathematical Society 
$S_{p}$. Conformally equivalent indefinite $C^{\infty}$ metrics determine the same ordered pair of null direction fields. Thus any Lorentz surface $\mathscr{L}=(S,[h])$ possesses a naturally ordered pair $X, Y$ of $C^{\infty}$ null direction fields.

Available anywhere on $S$ for an indefinite $C^{\infty}$ metric $h$ are local $C^{\infty}$ null coordinates $x, y$ in terms of which $h=2 F d x d y$ for some $C^{\infty}$ function $F \neq 0$. We assume that all null coordinates $x, y$ are chosen so as to respect not only the orientation on $S$, but the ordering of the null direction fields $X, Y$ for $h$ as well, so that $\partial / \partial x$ lies along $X$ and $\partial / \partial y$ along $Y$. (This amounts to assuming that $F>0$ above.) Since null coordinates $x, y$ for $h$ are also null for any $\hat{h} \sim h$, null coordinates are locally available anywhere on a Lorentz surface $\mathscr{L}=(S,[h])$.

We call Lorentz surfaces $\mathscr{L}=(S,[h])$ and $\hat{\mathscr{L}}=(\hat{S},[\hat{h}])$ conformally diffeomorphic (or $C^{\infty}$ conformally diffeomorphic) and write $\mathscr{L} \sim \hat{\mathscr{L}}$ iff there is an orientation-preserving $C^{\infty}$ diffeomorphism $F$ from $S$ onto $\hat{S}$ with $F^{*} \hat{h} \sim h$. The condition $F^{*} \hat{h} \sim h$ can be replaced by the requirement that $d F$ take the ordered pair of null direction fields $X, Y$ on $\mathscr{L}$ to the ordered pair of null direction fields $\hat{X}, \hat{Y}$ on $\hat{\mathscr{L}}$, or (equivalently) that $F$ take the family $\mathscr{Z}$ (resp. $\mathscr{Y}$ ) of maximal $C^{\infty}$ integral curves of $X$ (resp. $Y$ ) onto the family $\hat{\mathscr{X}}$ (resp. $\hat{Y}$ ) of maximal $C^{\infty}$ integral curves of $\hat{X}$ (resp. $\hat{Y}$ ). One can also replace the condition $F^{*} \hat{h} \sim h$ by the requirement that $F$ be locally expressible in terms of null coordinates $x, y$ for $h$ and $\hat{x}, \hat{y}$ for $\hat{h}$ by $C^{\infty}$ functions $\hat{x}=f(x)$ and $\hat{y}=g(y)$ with $f^{\prime}(x) g^{\prime}(y)>0$. However one frames the definition, $F$ is called a conformal diffeomorphism (or a $C^{\infty}$ conformal diffeomorphism) from $\mathscr{L}$ onto $\hat{\mathscr{L}}$.

We call Lorentz surfaces $\mathscr{L}=(S,[h])$ and $\hat{\mathscr{L}}=(\hat{S},[h]) C^{j}$ conformally diffeomorphic for $j=1,2, \ldots$ (resp. conformally homeomorphic) and write $\mathscr{L} \sim_{j} \hat{\mathscr{L}}$ (resp. $\mathscr{L} \sim_{0} \hat{\mathscr{L}}$ ) iff there is an orientation-preserving $C^{j}$ diffeomorphism (resp. an orientation-preserving homeomorphism) $F$ from $S$ onto $\hat{S}$ which takes each $\mathscr{X}$ curve to an $\hat{\mathscr{X}}$ curve and each $\mathscr{Y}$ curve to a $\hat{\mathscr{Y}}$ curve. Equivalently, one can require that $F$ be locally expressible in terms of null coordinates $x, y$ for $h$ and $\hat{x}, \hat{y}$ for $\hat{h}$ by $C^{j}$ (resp. $C^{0}$ ) functions $\hat{x}=f(x)$ and $\hat{y}=g(y)$ so that $f^{\prime}(x) g^{\prime}(y)>0$ (resp. so that $f$ and $g$ are both strictly increasing or both strictly decreasing). However one frames the definition, $F$ is called a $C^{j}$ conformal diffeomorphism (resp. a conformal homeomorphism) from $\mathscr{L}$ onto $\hat{\mathscr{L}}$.

To establish the claim in the title, we describe simply connected subsets of the Minkowski 2-plane $E_{1}^{2}$ which are conformally homeomorphic but not even $C^{1}$ conformally diffeomorphic. In addition, we describe for any $j=1,2, \ldots$ simply connected subsets of $E_{1}^{2}$ which are $C^{j}$ but not $C^{j+1}$ conformally diffeomorphic. Finally, we define a Lorentz surface which is conformally homeomorphic to a subset of $E_{1}^{2}$, but not even $C^{1}$ diffeomorphic to any subset of $E_{1}^{2}$.

\section{MORE BACKGROUND}

Following [1], we work with a conformal model of $E_{1}^{2}$ defined by using the metric $d x d y$ on the $(x, y)$-plane. We make no symbolic distinction between $E_{1}^{2}$ and the Lorentz surface $((x, y)$-plane, $[d x d y])$ which it determines. 

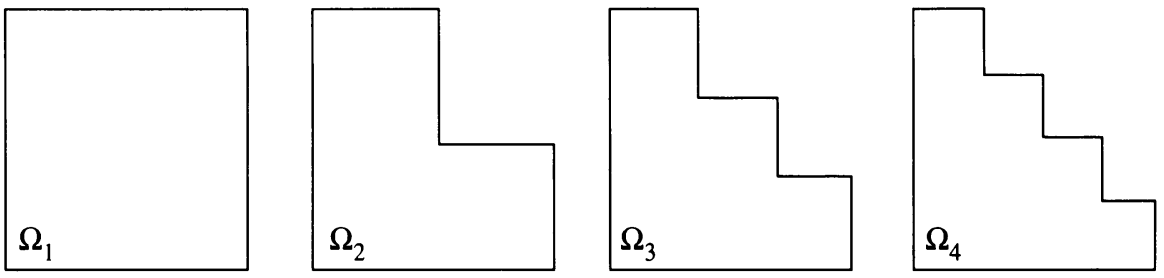

FIGURE 1

Suppose a Lorentz surface $\mathscr{L}=(S,[h])$ is simply connected (which means that $S$ is simply connected). Then $S$ is $C^{\infty}$ diffeomorphic to the 2-plane, and each curve in $\mathscr{X}$ or $\mathscr{Y}$ is a $C^{\infty}$ proper imbedding of the real line in $S$. Moreover, no two distinct curves in $\mathscr{X}$ (or in $\mathscr{Y}$ ) intersect, while an $\mathscr{X}$ curve intersects a $\mathscr{Y}$ curve in at most one point.

Any point on an arbitrary Lorentz surface $\mathscr{L}$ lies on exactly one curve from $\mathscr{X}$ and one from $\mathscr{Y}$ (modulo $C^{\infty}$ reparametrization). The span $\operatorname{sp}(\gamma)$ of a curve $\gamma$ in $\mathscr{X}$ (resp. $\mathscr{Y}$ ) is the set of all points on all curves in $\mathscr{Y}$ (resp. $\mathscr{X}$ ) which intersect $\gamma$. Note that $\gamma$ always lies in $\operatorname{sp}(\gamma)$. Though the $\operatorname{span} \operatorname{sp}(\gamma)$ for a curve in $\mathscr{X}$ or $\mathscr{Y}$ on a simply connected Lorentz surface must be conformally homeomorphic to a subset of $E_{1}^{2}$ (see [3]), we show in Theorem 3 that such a $\operatorname{span} \operatorname{sp}(\gamma)$ need not be $C^{1}$ conformally diffeomorphic to a subset of $E_{1}^{2}$.

As foundation for the results to follow, we describe in Lemma 1 a sequence of examples which confirm the observation in [1] that there are infinitely many simply connected Lorentz surfaces, no two of which are conformally homeomorphic. This contrasts sharply with the existence of exactly three conformally distinct simply connected Riemann surfaces.

Consider the regions $\Omega_{k}$ in the $(x, y)$-plane suggested by Figure 1. We think of $\Omega_{k}$ as a flight of $k$ steps for each $k=0,1, \ldots$. I.et $\mathscr{L}_{k}=\left(\Omega_{k},[d x d y]\right)$ so that curves in $\mathscr{X}$ (resp. $\mathscr{Y}$ ) are maximal horizontal (resp. vertical) line segments in $\Omega_{k}$. We have the following elementary fact.

Lemma 1. $\mathscr{L}_{k} \sim_{0} \mathscr{L}_{j}$ iff $k=j$.

Proof. The number of distinct sets $\operatorname{sp}(\gamma)$ on $S$ for all curves $\gamma$ in $\mathscr{X}$ on any one Lorentz surface $\mathscr{L}=(S,[h])$ is invariant under conformal homeomorphism. Since $\mathscr{L}_{k}$ has exactly $k$ distinct sets $\operatorname{sp}(\gamma)$ for all curves $\gamma$ in $\mathscr{Z}$, the lemma follows.

One further example deserves mention. Let $\mathscr{L}_{\infty}=\left(S_{\infty},[h]\right)$ where $S_{\infty}$ is the universal cover of the $(x, y)$-plane punctured at the origin and $h$ is the lift of the metric $d x d y$ to $S_{\infty}$. As shown in [1], $\mathscr{L}_{\infty}$ is not conformally homeomorphic to any subset of $E_{1}^{2}$.

\section{Results}

Let $\mathscr{L}=(R,[d x d y])$ where $R$ is the open region in the $(x, y)$-plane bounded by $x \equiv 1, y \equiv 0$ and the graph of a strictly increasing continuous function $y=f(x)$ on $[0,1]$ with $f(0)=0$ and $f(1)=1$. Let $\hat{\mathscr{L}}=(\hat{R},[d \hat{x} d \hat{y}])$ where $\hat{R}$ is the open triangular region in the $(\hat{x}, \hat{y})$-plane bounded by $\hat{x} \equiv 1, \hat{y} \equiv 0$ and $\hat{y}=\hat{x}$. Lemma 2 expands a result from [2]. It can be thought of as a $C^{k}$ "flattening lemma" for $k=0,1, \ldots, \infty$. 
Lemma 2. $\mathscr{L} \sim_{0} \hat{\mathscr{L}}$, and if $y=f(x)$ is also $C^{j}$ with $f^{\prime}(x)>0$ on $(0,1)$ for $j=1,2, \ldots, \infty$, then $\mathscr{L} \sim_{j} \hat{\mathscr{L}}$.

Proof. The map which takes any point $(x, y)$ in $R$ to the point $(\hat{x}, \hat{y})$ in $\hat{R}$ with $\hat{x}=f(x)$ and $\hat{y}=y$ establishes the result.

Keeping the same notation, we have the following result.

Theorem 1. If $y=f(x)$ is not $C^{k}$ with $f^{\prime}(x)>0$ on $(0,1)$ for some $k=$ $1,2, \ldots$, then $\mathscr{L} \sim_{k} \hat{\mathscr{L}}$ fails.

Proof. Assume that $y=f(x)$ is not $C^{k}$ with $f^{\prime}(x)>0$ on $(0,1)$, but that $F$ is a $C^{k}$ conformal diffeomorphism from $\mathscr{L}$ onto $\hat{\mathscr{L}}$. Then $F$ takes any horizontal (resp. vertical) line segment $\gamma$ in $R$ onto a horizontal (resp. vertical) line segment $F(\gamma)$ in $\hat{R}$, so that rectangles in $R$ with sides parallel to the coordinate axes are taken by $F$ to the same sort of rectangles in $\hat{R}$. Thus, if a horizontal line segment $\gamma_{1}$ in $R$ and a vertical line segment $\gamma_{2}$ in $R$ share an endpoint on the graph of $y=f(x)$ for $0<x<1$, then $F\left(\gamma_{1}\right)$ and $F\left(\gamma_{2}\right)$ must share an endpoint on $\hat{y}=\hat{x}$ for $0<\hat{x}<1$.

It follows that $F$ has a unique extension to a one-one continuous map $\bar{F}$ from the closure of $R$ which takes each endpoint of a maximal horizontal or vertical line segment $\gamma$ in $R$ to the corresponding endpoint of $F(\gamma)$, so that $(0,0)$ goes to $(0,0),(1,0)$ to $(1,0)$ and $(1,1)$ to $(1,1)$. For any point $p=\left(x_{0}, 0\right)$ with $0<x_{0}<1$, let $\bar{F}(p)=\left(\alpha\left(x_{0}\right), 0\right)$. For any point $p=\left(1, y_{0}\right)$ with $0<y_{0}<1$, let $\tilde{F}(p)=\left(1, \beta\left(y_{0}\right)\right)$. We conclude that the $C^{k}$ conformal diffeomorphism $F$ takes any point $(x, y)$ in $R$ to the point $(\hat{x}, \hat{y})$ in $\hat{R}$ with $\hat{x}=\alpha(x)$ and $\hat{y}=\beta(y)$, which means that $\alpha$ and $\beta$ are both $C^{k}$ with positive first derivative on $(0,1)$. In fact, $F$ is the restriction to $R$ of a $C^{k}$ conformal diffeomorphism from $(0,1) \times(0,1)$ onto $(0,1) \times(0,1)$ which takes $(x, y)$ to $(\alpha(x), \beta(y))$.

But since $\bar{F}$ takes the graph of $y=f(x)$ for $0<x<1$ onto the portion of $\hat{y}=\hat{x}$ with $0<\hat{x}<1$, we have

$$
\beta(y)=\alpha\left(f^{-1}(y)\right)
$$

so that $f^{-1}(y)=\alpha^{-1}(\beta(y))$ for $0<y<1$. As the composition of two $C^{k}$ functions with positive first derivatives, $f^{-1}$ must be $C^{k}$ with positive first derivative on $(0,1)$, a contradiction.

Together, Lemma 2 and Theorem 1 establish the claim in the title of the paper, and provide Lorentz surfaces $\mathscr{L}$ and $\hat{\mathscr{L}}$ which are $C^{j}$ but not $C^{j+1}$ conformally diffeomorphic for each $j=1,2, \ldots$. Formula (1) and the argument proving Theorem 1 show that any conformal homeomorphism $F$ from $\mathscr{L}=(R,[d x d y])$ onto $\hat{\mathscr{L}}=(\hat{R},[d \hat{x} d \hat{y}])$ is completely determined by the values of $\bar{F}$ on the line segment $y \equiv 0,0<x<1$.

Generally, any conformal homeomorphism $F: \mathscr{L} \rightarrow \hat{\mathscr{L}}$ between simply connected Lorentz surfaces $\mathscr{L}$ and $\hat{\mathscr{L}}$ extends uniquely to a homeomorphism $\tilde{F}$ from $\mathscr{L} \cup \partial \mathscr{L}$ onto $\hat{\mathscr{L}} \cup \partial \hat{\mathscr{L}}$ where $\partial \mathscr{L}$ and $\partial \hat{\mathscr{L}}$ are the conformal boundaries of $\mathscr{L}$ and $\hat{\mathscr{L}}$, respectively, defined by Kulkarni in [1]. Typically, $F$ is completely determined by the values of $\tilde{F}$ on just a portion of $\partial \mathscr{L}$. The next example we study further illustrates this phenomenon. 

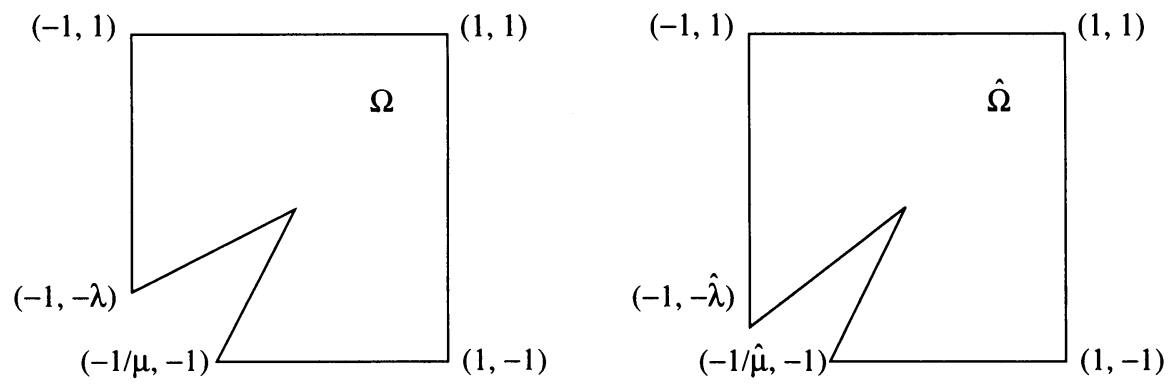

FIGURE 2
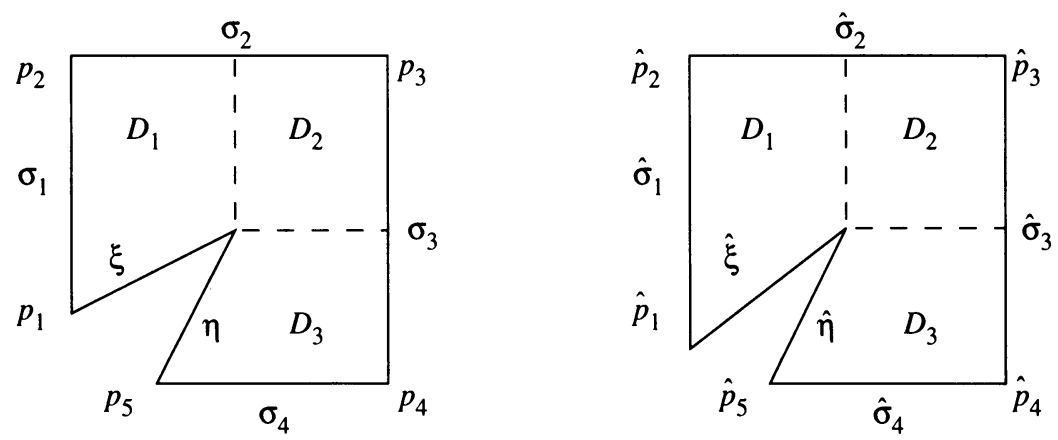

Figure 3

Let $\mathscr{L}=(\Omega,[d x d y])$ and $\hat{\mathscr{L}}=(\hat{\Omega},[d \hat{x} d \hat{y}])$ where $\Omega$ and $\hat{\Omega}$ are the subsets of the $(x, y)$-plane and the $(\hat{x}, \hat{y})$-plane, respectively, shown in Figure 2 , with $0<\lambda<1<\mu$ and $0<\hat{\lambda}<1<\hat{\mu}$. We have the following observation.

Lemma 3. $\mathscr{L} \sim_{0} \hat{\mathscr{L}}$.

Proof. Let $\hat{y}=g(y)$ be continuous on $[-\lambda, 1]$ and $C^{\infty}$ on $(-\lambda, 1)$ with $g^{\prime}(y)>0$ on $(-\lambda, 1), g(y)=(\hat{\lambda} / \lambda) y$ on $[-\lambda, 0]$ and $g(1)=1$. Let $\hat{x}=$ $\varphi(x)$ be continuous on $[-1 / \mu, 1]$ and $C^{\infty}$ on $(-1 / \mu, 1)$ with $\varphi^{\prime}(x)>0$ on $(-1 / \mu, 1), \varphi(x)=(\mu / \hat{\mu}) x$ on $[-1 / \mu, 0]$ and $\varphi(1)=1$. Let $f(x)$ be continuous on $[-1,1]$ and $C^{\infty}$ on $(-1,1)$ with $f^{\prime}(x)>0$ on $(-1,1)$, $f(x)=x$ on $[-1,0]$ and $f(x)=\varphi(x)$ on $[0,1]$. Use the intersection of $\Omega$ with the positive $x$-axis to divide $\Omega$ into two relatively closed sets $R_{1}$ and $R_{2}$, with $y \leq 0$ throughout $R_{2}$. Define the homeomorphism $F$ from $\Omega$ onto $\hat{\Omega}$ by taking $\hat{x}=f(x), \hat{y}=g(y)$ in $R_{1}$ and $\hat{x}=\varphi(x), \hat{y}=y$ in $R_{2}$. Note that $F$ is a $C^{\infty}$ diffeomorphism except along the positive $x$ and $y$ axes.

Now use Figure 3 to define the open line segments $\xi, \eta, \hat{\xi}, \hat{\eta}, \sigma_{i}$ and $\hat{\sigma}_{i}$ for $i=1, \ldots, 4$, the points $p_{i}$ and $\hat{p}_{i}$ for $i=1, \ldots, 5$ and the closed sets $D_{1}, D_{2}$ and $D_{3}$ (resp. $\hat{D}_{1}, \hat{D}_{2}$ and $\hat{D}_{3}$ ) whose union is the closure of $\Omega$ (resp. $\hat{\Omega}$ ). Any conformal homeomorphism $F$ from $\mathscr{L}$ onto $\hat{\mathscr{L}}$ takes horizontal (resp. vertical) line segments in $\Omega$ onto horizontal (resp. vertical) line segments in $\hat{\Omega}$. Thus such an $F$ takes the maximal line segment $y \equiv \varepsilon>0$ in $\Omega$ (which approaches the two maximal line segments with $y \equiv 0$ in $\Omega$ as $\varepsilon \rightarrow 0$ ) to a maximal line segment $\hat{y} \equiv \hat{\varepsilon}>0$ in $\hat{\Omega}$ (which approaches the two 
maximal line segments with $\hat{y} \equiv 0$ in $\hat{\Omega}$ as $\hat{\varepsilon} \rightarrow 0$ ). It follows that $F$ takes $\Omega \cap\{y>0\}$ onto $\hat{\Omega} \cap\{\hat{y}>0\}$ and similarly that $F$ takes $\Omega \cap\{x>0\}$ onto $\hat{\mathbf{\Omega}} \cap\{\hat{x}>0\}$.

Given a conformal homeomorphism $F$ from $\mathscr{L}$ onto $\hat{\mathscr{L}}$, we conclude that $F$ extends to a homeomorphism $\bar{F}$ on the closure of $\Omega$ which takes each endpoint of any maximal horizontal or vertical line segment $\gamma$ in $\Omega$ to the corresponding endpoint of $F(\gamma)$, so that $\bar{F}$ takes $(0,0)$ to $(0,0), p_{i}$ to $\hat{p_{i}}$ for $i=1, \ldots, 5, \sigma_{i}$ to $\hat{\sigma}_{i}$ for $i=1, \ldots, 4, D_{i}$ to $\hat{D}_{i}$ for $i=1, \ldots, 3, \xi$ to $\hat{\xi}$ and $\eta$ to $\hat{\eta}$. This yields the following results.

Lemma 4. The values of $\bar{F}$ on the closure of $\Omega$ are determined by its values on $\sigma_{1}$ and $\sigma_{4}$.

Proof. Let $\bar{F}$ take $(x, y)$ to $(\hat{x}, \hat{y})$. Suppose $\hat{y}=g(y)$ on $\sigma_{1}, \hat{x}=f(x)$ on $\sigma_{2}, \hat{y}=\gamma(y)$ on $\sigma_{3}$ and $\hat{x}=\varphi(x)$ on $\sigma_{4}$. Since $\bar{F}$ takes horizontal intervals in $\Omega$ to horizontal intervals in $\hat{\Omega}, \hat{y}=g(y)$ throughout $D_{1} \cup D_{2}$. Since $F$ takes vertical intervals in $\Omega$ to vertical intervals in $\hat{\Omega}, \hat{x}=\varphi(x)$ throughout $D_{2} \cup D_{3}$. Thus $f(x)=\varphi(x)$ for $x \geq 0$ and $\gamma(y)=g(y)$ for $y \geq 0$. We need only show that $g(y)$ determines $f(x)$ for $x<0$ and that $\varphi(x)$ determines $\gamma(y)$ for $y<0$. But since $\hat{y}=\hat{\lambda} \hat{x}$ must hold on $\xi$ where $y=\lambda x, \bar{F}$ on $\xi$ must take $(x, y)$ to $(\hat{x}, g(y))$ with $g(y)=\hat{\lambda} \hat{x}$, so that $\hat{x}=g(\lambda x) / \hat{\lambda}$ for all $x<0$ and by continuity, for all $x \leq 0$ in $D_{1}$. Similarly, since $\hat{y}=\hat{\mu} \hat{x}$ on $\eta$ where $y=\mu x, \bar{F}$ on $\eta$ takes $(x, y)$ to $(\varphi(x), \hat{y})$ with $\hat{y}=\hat{\mu} \varphi(x)$ so that $\hat{y}=\hat{\mu} \varphi(y / \mu)$ for all $y \leq 0$ in $D_{3}$.

Theorem 2. $\mathscr{L} \sim_{1} \hat{\mathscr{L}}$ (and $\mathscr{L} \sim \hat{\mathscr{L}}$ ) iff $\lambda \hat{\mu}=\hat{\lambda} \mu$.

Proof. Suppose $F$ is a $C^{1}$ conformal diffeomorphism from $\mathscr{L}$ onto $\hat{\mathscr{L}}$. Let $\bar{F}$ be the extension of $F$ to a homeomorphism from the closure of $\Omega$ onto the closure of $\hat{\Omega}$. Using the notation in the proof of Lemma $4, f(x)$ must be $C^{1}$ on $(-1,1), \varphi(x)$ must be $C^{1}$ on $(-1 / \mu, 1), g(y)$ must be $C^{1}$ on $(-\lambda, 1)$ and $\gamma(y)$ must be $C^{1}$ on $(-1,1)$. These claims hold since $\hat{x}=f(x)$ and $\hat{y}=g(y)$ in the interior of $D_{1} \cup D_{2}$ where $F=\bar{F}$ is $C^{1}$ and every $x$ value in $(-1,1)$ and $y$ value in $(-\lambda, 1)$ is represented, while $\hat{x}=\varphi(x)$ and $\hat{y}=\gamma(y)$ in the interior of $D_{2} \cup D_{3}$ where $F=\bar{F}$ is $C^{1}$ and every $y$ value in $(-1,1)$ and $x$ value in $(-1 / \mu, 1)$ is represented.

As shown in the proof of Lemma $4, \hat{x}=g(\lambda x) / \hat{\lambda}$ and $\hat{x}=f(x)$ in $D_{1}$ while $\hat{y}=\hat{\mu} \varphi(y / \mu)$ and $y=\gamma(y)$ in $D_{3}$. Thus

$$
f(x)=g(\lambda x) / \hat{\lambda}
$$

for $x \leq 0$ while

$$
\gamma(y)=\hat{\mu} \varphi(y / \mu)
$$

for $y \leq 0$. Since $f(x)=\varphi(x)$ for $x \geq 0$ and $g(y)=\gamma(y)$ for $y \geq 0$, the chain rule applied to (2) and (3) gives

$$
g^{\prime}(0)=\gamma^{\prime}(0)=(\hat{\mu} / \mu) \varphi^{\prime}(0)=(\hat{\mu} / \mu) f^{\prime}(0)=(\hat{\mu} \lambda / \mu \hat{\lambda}) g^{\prime}(0) .
$$

Since $F$ is a $C^{1}$ diffeomorphism, $g^{\prime}(0) \neq 0$ in (4), so $\lambda \hat{\mu}=\hat{\lambda} \mu$ as claimed. In case $F$ is $C^{\infty}, f(x), g(y), \varphi(x)$ and $\gamma(y)$ are all $C^{\infty}$. Taking higher derivatives, one gets $g^{(n)}(0)=\varphi^{(n)}(0)=0$ for $n=2, \ldots$, since $\lambda \neq \mu$. 
Conversely, suppose $\Omega$ and $\hat{\Omega}$ in Figure 2 have $\lambda \hat{\mu}=\hat{\lambda} \mu$. Construct an orientation-preserving homeomorphism $\bar{F}$ from the closure of $\Omega$ onto the closure of $\hat{\Omega}$ which restricts to a $C^{\infty}$ diffeomorphism $F$ on $\Omega$ taking horizontal (resp. vertical) line segments to horizontal (resp. vertical) line segments as follows. Take a continuous $g(y)$ on $[-\lambda, 1]$ with $g(-\lambda)=-\hat{\lambda}$, $g(0)=0$ and $g(1)=1$ which is $C^{\infty}$ with $g^{\prime}(y)>0$ on $(-\lambda, 1)$ while $g^{(n)}(0)=0$ for $n=2, \ldots$, and take a continuous $\varphi(x)$ on $[-1 / \mu, 1]$ with $\varphi(-1 / \mu)=-1 / \hat{\mu}, \varphi(0)=0$ and $\varphi(1)=1$ which is $C^{\infty}$ with $\varphi^{\prime}(x)>0$ on $(-1 / \mu, 1)$ with $\varphi^{\prime}(0)=\mu g^{\prime}(0) / \hat{\mu}=\lambda g^{\prime}(0) / \hat{\lambda}$ and $\varphi^{(n)}(0)=0$ for $n=2, \ldots$. Set $f(x)=g(\lambda x) / \hat{\lambda}$ for $x \leq 0, \gamma(y)=\hat{\mu} \varphi(y / \mu)$ for $y \leq 0, f(x)=\varphi(x)$ for $x \geq 0$ and $\gamma(y)=g(y)$ for $y \geq 0$. Define $\bar{F}$ on $\bar{\Omega}$ by taking $\hat{x}=f(x)$, $\hat{y}=g(x)$ on $D_{1} \cup D_{2}$ and $\hat{x}=\varphi(x), \hat{y}=\gamma(y)$ on $D_{2} \cup D_{3}$. Since $\lambda \hat{\mu}=\hat{\lambda} \mu$, (4) holds. By construction, the restriction $F$ of $\bar{F}$ to $\Omega$ yields a conformal diffeomorphism from $\mathscr{L}=(\Omega,[d x d y])$ onto $\hat{\mathscr{L}}=(\hat{\Omega},[d \hat{x} d \hat{y}])$.

One can easily find values $\lambda, \mu, \hat{\lambda}$ and $\hat{\mu}$ with $0<\lambda<1<\mu, 0<\hat{\lambda}<$ $1<\hat{\mu}$ and $\lambda \hat{\mu} \neq \hat{\lambda} \mu$. By Lemma 3 and Theorem 2, small variations in $\hat{\lambda}$ with $\hat{\mu}$ held fixed yield uncountably many conformally homeomorphic Lorentz surfaces, no two of which are $C^{1}$ conformally diffeomorphic.

Our final result involves the set $D$ in the $(x, y)$-plane obtained by removing all points on $y \equiv 0$ with $x \geq 0$ from the region $(-1,1) \times(-1,1)$. Denote by $S$ the set $D$ with its usual $C^{\infty}$ structure. Denote by $\hat{S}$ the set $D$ with the $C^{\infty}$ structure determined by the chart $x_{1}=x^{3}, y_{1}=y$ on $U_{1}=D \cap\{y>0\}$ and the chart $x_{2}=x, y_{2}=y$ on $U_{2}=D \cap(\{y<0\} \cup\{x<0\})$. Let $\mathscr{L}=(S,[d x d y])$ and $\hat{\mathscr{L}}=(\hat{S},[h])$ where $h=\varphi_{1} d x_{1} d y_{1}+\varphi_{2} d x_{2} d y_{2}$ for a $C^{\infty}$ partition of unity $\varphi_{1}, \varphi_{2}$ subordinate to the covering of $\hat{S}$ by $U_{1}$ and $U_{2}$. The identity map from $S$ onto $\hat{S}$ establishes that $\mathscr{L} \sim_{0} \hat{\mathscr{L}}$. Still, we have the following result.

Theorem 3. $\hat{\mathscr{L}}$ is not $C^{1}$ conformally diffeomorphic to any subset of $E_{1}^{2}$.

Proof. Let $F$ be a $C^{1}$ conformal diffeomorphism of $\hat{\mathscr{L}}$ into $E_{1}^{2}=((u, v)$ plane, $[d u d v])$. With no loss of generality, assume that $v>0$ at $F(p)$ for any $p$ in $U_{1}$ and that $F$ takes the portion of $y \equiv 0$ in $\hat{\mathscr{L}}$ to a line segment along $v \equiv 0$ with its right endpoint at the origin. Let $\gamma^{+}$and $\gamma^{-}$be the portions of the lines $y \equiv 1 / 2$ and $y \equiv-1 / 2$, respectively, in $\hat{\mathscr{L}}$. Then $F$ must take the rectangular region $\{x<0\}$ in $\hat{\mathscr{L}}$ onto a region $(a, 0) \times(c, d)$ in $E_{1}^{2}$ where $-\infty \leq a<0,-\infty \leq c<0$ and $\infty \geq d>0$. Use the $u$ values on $F\left(\gamma^{+}\right)$and $F\left(\gamma^{-}\right)$to provide $C^{1}$ regular parametrizations of $\gamma^{+}$ and $\gamma^{-}$over intervals $\left(a, b^{+}\right)$and $\left(a, b^{-}\right)$, respectively, with $\infty \geq b^{+}>0$ and $\infty \geq b^{-}>0$. Assume that $b^{+} \geq b^{-}$. (A similar argument applies if $b^{-}>b^{+}$.) Then the mapping of $F\left(\gamma^{-}\right)$into $F\left(\gamma^{+}\right)$by matching $u$ values is a $C^{\infty}$ diffeomorphism. Since $F^{-1}$ takes vertical line segments to vertical line segments, this means that the mapping of $\gamma^{-}$into $\gamma^{+}$by matching $u$ parameter values must be a $C^{1}$ diffeomorphism taking any point on $\gamma^{-}$with $x<0$ to the point on $\gamma^{+}$with the same $x$ value. But then, for some $b$ in $(0,1]$ the identity map on $(-1,0]$ can be extended to a $C^{1}$ diffeomorphism from the interval $(-1,1)$ with the usual differentiable structure onto an interval $(-1, b)$ 
with the differentiable structure determined by the chart coordinate $x_{1}=x^{3}$. This is a contradiction.

\section{REFERENCES}

1. R. Kulkarni, An analogue of the Riemann mapping theorem for Lorentz metrics, Proc. Roy. Soc. London Ser. A 401 (1985), 117-130.

2. T. Weinstein, Inextendible conformal realizations of Lorentz surfaces in Minkowski 3-space, Michigan Math. J. 40 (1993), 545-559.

3. __ An Introduction to Lorentz surfaces, Expositions in Math., de Gruyter, Berlin and New York (submitted).

Department of Mathematics, Rutgers University, New Brunswick, New Jersey 08903

E-mail address: rsmyth@math.rutgers .edu

E-mail address: tilla@math.rutgers.edu 